\title{
Bone Healing in Adolescents with Bilateral L5 Spondylolysis with and without Preexisting Contralateral Terminal Spondylolysis
}

\author{
Fumihiko Eto ${ }^{1,2}$, Masaki Tatsumura ${ }^{1}$, Hisanori Gamada ${ }^{1}$, Shun Okuwaki ${ }^{1}$, Masao Koda ${ }^{2}$, Masashi Yamazaki $^{2}$ \\ ${ }^{I}$ Department of Orthopaedic Surgery and Sports Medicine, Tsukuba University Hospital Mito Clinical Education and Training Center, \\ Mito Kyodo General Hospital, Mito, Japan \\ ${ }^{2}$ Department of Orthopaedic Surgery, Faculty of Medicine, University of Tsukuba, Tsukuba, Japan
}

Study Design: Retrospective cohort study.

Purpose: To examine bone healing with conservative treatment in cases of adolescent bilateral $L 5$ spondylolysis.

Overview of Literature: We classified bilateral L5 spondylolysis, which is the most affected spinal level, by fracture stage and aimed to compare the bone healing rate according to the fracture stage and evaluate the presence of a preexisting contralateral terminal fracture at the diagnosis of fresh spondylolysis.

Methods: We evaluated 48 patients (38 boys and 10 girls) with bilateral L5 spondylolysis diagnosed during or before high school. L5 spondylolysis was classified into two groups: fresh group (bilateral fresh spondylolysis cases), and terminal group (cases wherein one side had fresh spondylolysis and the contralateral side had terminal spondylolysis). We investigated the age of examination and bone healing rate in both groups. We investigated progressive-stage lesions and bone healing rate with or without progressive-stage lesions.

Results: The bone healing rate in the fresh group was significantly higher than that in the terminal group $72.0 \%$ vs. $26.1 \%, p=0.003$ ). In both the groups, the bone healing rate was significantly higher in patients without progressive-stage lesions than in those with progressive-stage lesions.

Conclusions: Bone healing of progressive-stage fresh spondylolysis was not achieved by conservative treatment when contralateral terminal spondylolysis was present in adolescents with bilateral L 5 spondylolysis. Our results suggest that bilateral L 5 spondylolysis treatment strategies must be determined based on the combination of the stages present.

Keywords: Spondylolysis; Bone remodeling; Conservative treatment

\section{Introduction}

Lumbar spondylolysis is a stress fracture of the pars interarticularis and that typically occurs in adolescent athletes
[1]. Kaneko et al. [2] reported that 33\% of young patients that complained of sports-related low back pain lasting more than 7 days had lumbar stress injuries. The L5 pars interarticularis is the spinal level most affected and sus-

\footnotetext{
Received Jun 21, 2020; Revised Aug 14, 2020; Accepted Aug 15, 2020

Corresponding author: Fumihiko Eto

Tsukuba University Hospital Mito Clinical Education and Training Center, Mito Kyodo General Hospital, 3-2-7 Miyamachi, Mito, Ibaraki 310-0015, Japan

Tel: +81-29-231-2371, Fax: +81-29-221-5137, E-mail: f.etoh@tsukuba-seikei.jp
} 
ceptible to mechanical stress [3].

Recent diagnostic advances in magnetic resonance imaging (MRI) have resulted in early diagnosis of spondylolysis [4]. Current treatment strategies suggest that early diagnosis, coupled with conservative treatment for lumbar spondylolysis, is a way to achieve successful bone healing. Sometimes, bone healing is not achieved even with early diagnosis and conservative treatment.

Patients diagnosed with terminal spondylolysis at first consultation cannot achieve successful bone healing. When spondylolysis progresses to terminal-stage, inflammation may first occur at the site of pseudoarthrosis and then spread to the adjoining facet joints and synovitis, causing low back pain [5]. There is a possibility that this process progresses to lumbar spondylolisthesis, where a vertebra slips from its location to rest on the lower bone. There have been some reports that the pathological stage on the contralateral side can affect bone healing [6-8], but no reports have exclusively examined L5 spondylolysis.

For this study, we aimed to evaluate bone healing rates in L5 spondylolysis comparing the fracture stages present.

\section{Materials and Methods}

Using both MRI and computed tomography (CT), we identified students diagnosed for the first time with bilateral L5 spondylolysis. Patients with pseudoarthrosis on both sides at the first consultation were excluded. We constructed a cohort of 48 patients ( 38 boys and 10 girls) who were seen at our facility between April 2015 and March 2019. The mean age of the patients was 13.5 years (range, 8-17 years). All patients were students who were active in both sports and club activities. The most popular sport activity was soccer, with 13 patients (27\%), followed by baseball with 11 patients (23\%). No patient had any history of medical or spinal disorders. Informed consent was obtained from all participants in this study. The study was performed in accordance with the Declaration of Helsinki and approved by the Institutional Review Board of Mito Kyodo General Hospital (approval no., 19-44).

Stages were classified according to previous reports using both MRI and CT at the first consultation [9]. An MRI showing bone marrow edema with CT indicating visible hairline fracture was classified as an early-stage. Alternatively, an MRI showing bone marrow edema and CT indicating clear bone gap was classified as a progressive-stage. An MRI showing the absence of bone marrow edema with
CT indicating bone defect was classified as the terminalstage. Our conservative treatment protocol for lumbar spondylolysis, other than terminal-stage, was for patients to wear a semi-rigid lumbosacral back brace to limit trunk extension, refrain from participating in sports, and undergo physiotherapy [9]. Since the disappearance of bone marrow edema on MRI has been reported to indicate the loss of a fracture healing mechanism [10,11], an MRI was performed every 1-2 months until the bone marrow edema around the pedicle resolved on short tau inversion recovery images. When bone marrow edema improved on both sides, patients could remove the brace and return to playing sports. After brace removal, a CT was performed to determine bone healing. Bone healing was confirmed if bone continuity had been achieved, even in part, or if a bone bridge was observed on CT, even if a fracture line remained. In contrast, a pseudoarthrosis was diagnosed when no bone continuity was observed on CT, even when bone marrow edema was no longer clear on MRI.

Fresh spondylolysis was defined as early- and progressive-stage lesions with potential bone healing, and terminal spondylolysis was defined as terminal-stage lesions with no potential for bone healing. We classified L5 spondylolysis into two groups: the fresh group included patients with bilateral fresh spondylolysis, and the terminal group included patients with fresh spondylolysis on one side and terminal spondylolysis on the contralateral side (Fig. 1).

The treatment period was defined as the time between diagnosis of fresh spondylolysis and the improvement of
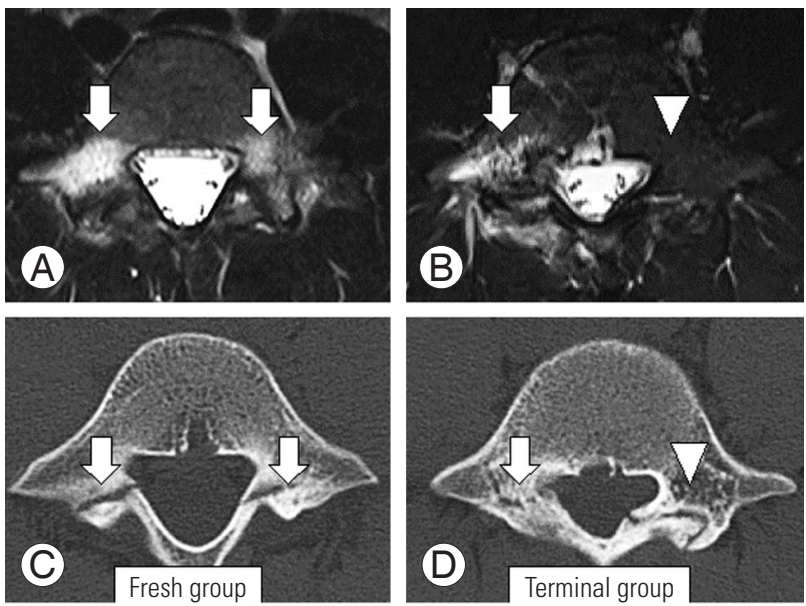

Fig. 1. Axial views on magnetic resonance imaging $(\mathbf{A}, \mathbf{B})$ and computed tomography $(\mathbf{C}, \mathbf{D})$ in each group. Arrow and arrowhead indicate fresh and terminal spondylolysis, respectively. 
bone marrow edema on both sides via MRI, regardless of bone union. The mean treatment period was 3.7 months.

We assessed the age of examination and bone healing rate in both groups. Within the fresh spondylolysis group, we stratified bone healing rates for cases with progressivestage lesions on one or both sides and cases with no progressive-stage lesions on either side, in other words, only those patients with early-stage lesions.

All values are expressed as mean \pm standard deviation. Differences in age were evaluated using Welch's $t$-test, and Fisher's exact test was used to compare the bone healing rate. We considered a value of $p<0.05$ to be statistically significant.

\section{Results}

There were 25 patients in the fresh group and 23 patients in the terminal group. The mean ages in the fresh group and the terminal group were 13.2 and 13.9 years, respectively, with no significant difference $(p=0.25)$.

In the fresh group, none of the patients achieved bone healing on only one side. Bone healing was achieved in 18 patients in the fresh group and six patients in the terminal group. The rate of bone healing was significantly higher in the fresh group than in the terminal group (72.0 versus $26.1 \%, p=0.003$ ) (Table 1).

Table 2 presents the number of cases with each combination of the stages on each side. In the fresh group, there were nine patients with only early-stage lesions and 16 patients with progressive-stage lesions. Bone healing was achieved in nine patients (100\%) and nine patients (56.3\%), respectively. Therefore, even with bilateral fresh spondylolysis, the bone healing rate significantly decreased when there were progressive-stage lesions ( $p=0.03$ ). There were 10 patients with early-stage lesions,

Table 1. Bone healing rate in each group

\begin{tabular}{|c|c|c|c|}
\hline \multirow{2}{*}{ Characteristic } & \multicolumn{2}{|c|}{ Group } & \multirow{2}{*}{$p$-value } \\
\hline & Fresh & Terminal & \\
\hline No. of patients & 25 & 23 & \\
\hline Age (yr) & $13.2 \pm 1.7$ & $13.9 \pm 2.1$ & 0.25 \\
\hline Bone healing & 18 & 6 & \\
\hline Bone healing rate (\%) & 72 & 26.1 & $0.003^{*}$ \\
\hline
\end{tabular}

Values are presented as number or mean \pm standard deviation, unless otherwise stated.

" $p<0.05$. and 13 patients with progressive-stage lesions in the terminal group. Bone healing was achieved in six patients $(60 \%)$ and no patients $(0 \%)$, respectively, and a significant difference in the bone healing rate was also observed $(p=0.002)$. There was a significant difference in the bone healing rate between the fresh and terminal groups with progressive-stage lesions ( $56.3 \%$ versus $0 \%, p=0.001$ ) but not in patients with only early-stage lesions (100 versus $60 \%, p=0.09$ ) (Fig. 2).

\section{Case 1: fresh group}

A 14-year-old boy developed low back pain during baseball practice and visited Mito Kyodo General Hospital 2

Table 2. Numbers of cases based on the combination of spondylolysis stages

\begin{tabular}{lccc}
$\begin{array}{l}\text { Stage of spondylolysis } \\
\text { (one side-contralateral } \\
\text { side) }\end{array}$ & Total & & \multicolumn{2}{c}{ Bone healing } \\
\cline { 3 - 4 } $\begin{array}{l}\text { Early-early } \\
\text { Early-progressive }\end{array}$ & 9 & 9 & 9 \\
\hline Progressive-progressive & 7 & 6 & Contralateral side \\
\hline Early-terminal & 9 & 3 & 6 \\
\hline Progressive-terminal & 10 & 6 & 0 \\
\hline
\end{tabular}

Values are presented as numbers.

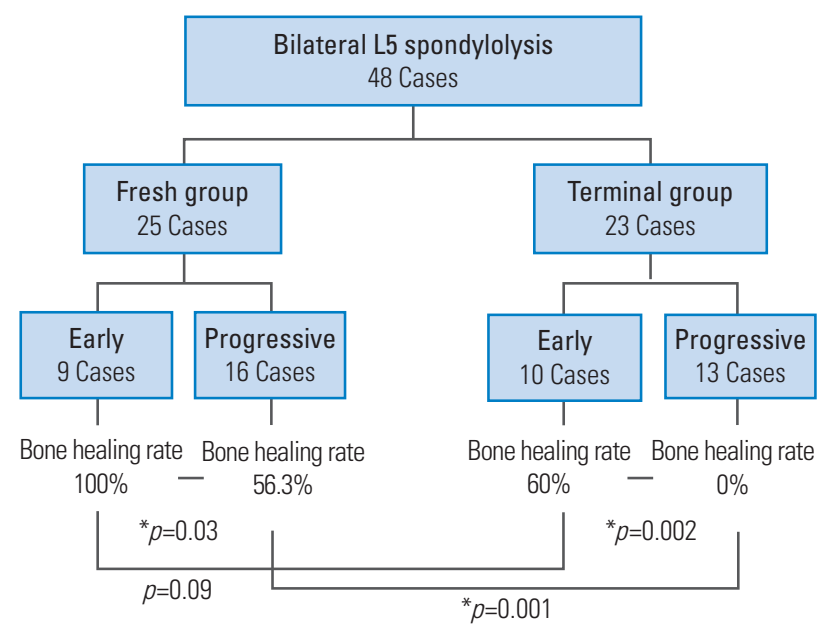

Fig. 2. Bone healing rate with or without progressive-stage lesions was investigated in each group. Bone healing rates were compared in each group and between the two groups. Early means there were only early-stage lesions in fresh spondylolysis. Progressive means there were progressive-stage lesions on one or both sides. 

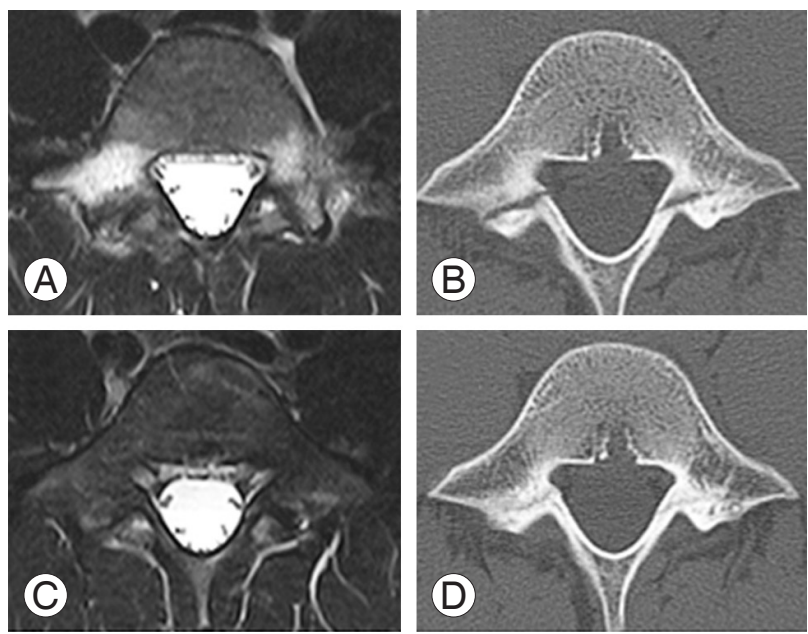

Fig. 3. Fresh group: the case of a 14-year-old baseball player. (A) Magnetic resonance imaging revealed bilateral bone marrow edema at the first examination. (B) An axial computed tomography scan revealed a progressive-stage fracture on the right side and an early-stage fracture on the left side. (C, D) Three months later, we saw improved bone marrow edema and bone healing was achieved.

months later. MRI revealed bone marrow edema on both sides of L5 (Fig. 3A). We diagnosed lumbar spondylolysis on axial CT that revealed progressive-stage lesion on the right side and early-stage lesion on the left side (Fig. 3B). The patient rested, began wearing a semi-rigid lumbosacral back brace, and started physiotherapy. After 3 months of conservative treatment, MRI showed improved bone marrow edema on both sides (Fig. 3C) and CT revealed bone healing (Fig. 3D).

\section{Case 2: terminal group}

A 17-year-old boy developed low back pain during soccer practice and visited Mito Kyodo General Hospital a month later. MRI revealed bone marrow edema on the right side of L5 (Fig. 4A). We diagnosed lumbar spondylolysis with an axial CT that showed an early-stage lesion on the right side, and a terminal-stage lesion on the left side (Fig. 4B). He rested, began wearing a semi-rigid lumbosacral back brace, and started physiotherapy. After 4 months of conservative treatment, MRI revealed that the bone marrow edema on the right side had improved (Fig. 4C). However, bone healing was not seen on a CT scan, and he was diagnosed with pseudoarthrosis (Fig. 4D).
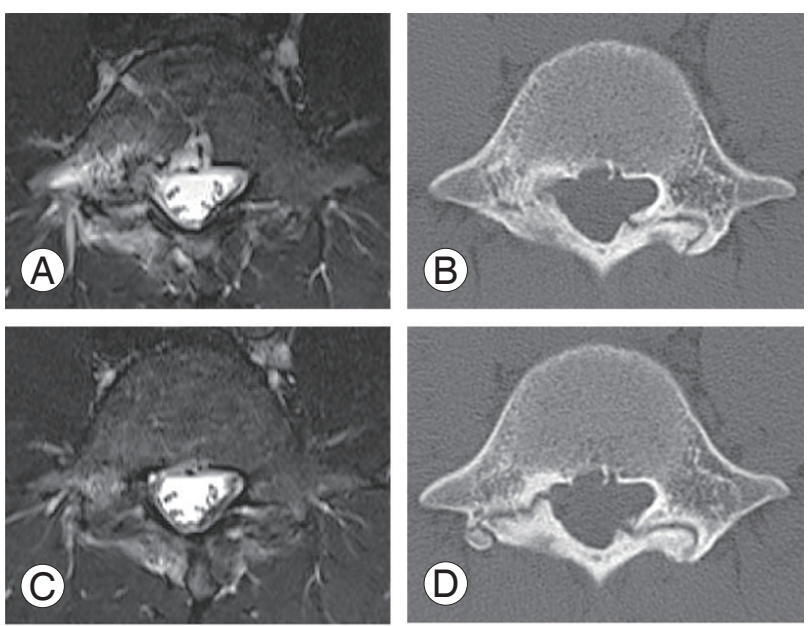

Fig. 4. Terminal group: the case of a 17-year-old soccer player. (A) Magnetic resonance imaging revealed bone marrow edema on the right side at the first examination. (B) An axial computed tomography scan revealed an early-stage fracture on the right side and a terminal-stage fracture on the left side. (C, D) Four months later, bone marrow edema had disappeared, but bone healing was not achieved.

\section{Discussion}

In the present study, the bone healing rate of bilateral L5 spondylolysis differed depending on the combination of fracture stages present. Conservative treatment resulted in a significantly higher rate of bone healing in patients with bilateral fresh spondylolysis compared to those with fresh spondylolysis on one side and terminal spondylolysis on the contralateral side. In particular, bone healing of progressive-stage fresh spondylolysis was not achieved by conservative treatment when contralateral terminal spondylolysis was present. Even with bilateral fresh spondylolysis, the bone healing rate decreased when there were progressive-stage lesions.

In a normal lumbar spine, the posterior surface of the vertebral body, pedicles, and lamina form an annular structure. When fresh spondylolysis occurs on only one side, the continuity of the annular structure is maintained and there is no instability. However, continuation of exercise without treatment is expected to decrease the ability to heal for the condition. It has been reported that a unilateral pars defect could increase the risk of a spondylolysis on the contralateral side [12]. If one side progresses to terminal spondylolysis, it subsequently causes bilateral spondylolysis.

Where bone healing was achieved with conservative treatment, Yamazaki et al. [6] reported that bone healing 
rates where the contralateral side was progressive-stage and terminal-stage were $16.9 \%$ and $3.4 \%$, respectively. Fujii et al. [8] reported that the bone healing rate without contralateral defects or with contralateral early-stage spondylolysis was significantly higher than that for those with contralateral progressive or terminal-stage spondylolysis. These data suggest that the lumbar spondylolysis stage on the contralateral side is involved in the bone healing process.

In the present study, we hypothesized that individuals with bilateral L5 spondylolysis had different bone healing rates depending on whether they had progressive or terminal-stage lesions, had mechanical instability and a decreased bone healing rate because of disruption of bone continuity.

Therefore, we propose that conservative treatment is not indicated in patients with progressive-staged fresh spondylolysis on one side and terminal spondylolysis on the contralateral side because bone healing of fresh spondylolysis cannot be achieved. In addition, it should be recognized that when selecting conservative treatment, even with bilateral fresh spondylolysis, the bone healing rate of progressive-stage lesions is significantly decreased.

To prevent the progression to terminal-stage, it would be desirable to diagnose lumbar spondylolysis in adolescents at an early stage, and perform conservative treatment aimed at bone healing. However, with bilateral L5 spondylolysis, bone healing rates in conservative treatment were different depending on the combination of lumbar spondylolysis stages. Therefore, subsequent spondylolysis treatment strategies should be determined based on CT and MRI imaging evaluations.

\section{Conclusions}

In the present study regarding bilateral L5 spondylolysis in adolescents, bone healing of progressive-stage fresh spondylolysis was not achieved by conservative treatment when contralateral terminal spondylolysis was present. Bone healing was likely to be achieved if there were only early-stage lesions on both sides. Our data demonstrates that it is important to evaluate the combinations of lumbar spondylolysis via initial CT and MRI to determine subsequent treatment strategies.

\section{Conflict of Interest}

No potential conflict of interest relevant to this article was reported.

\section{Acknowledgments}

We thank Miki Omori, medical clerk at Mito Clinical Education and Training Center, University of Tsukuba Hospital, and Mito Kyodo General Hospital, for her valuable contribution to data analysis.

\section{References}

1. Wiltse LL, Widell EH Jr, Jackson DW. Fatigue fracture: the basic lesion is inthmic spondylolisthesis. J Bone Joint Surg Am 1975;57:17-22.

2. Kaneko H, Murakami M, Nishizawa K. Prevalence and clinical features of sports-related lumbosacral stress injuries in the young. Arch Orthop Trauma Surg 2017;137:685-91.

3. Goda Y, Sakai T, Sakamaki T, Takata Y, Higashino K, Sairyo K. Analysis of MRI signal changes in the adjacent pedicle of adolescent patients with fresh lumbar spondylolysis. Eur Spine J 2014;23:1892-5.

4. Sairyo K, Katoh S, Takata Y, et al. MRI signal changes of the pedicle as an indicator for early diagnosis of spondylolysis in children and adolescents: a clinical and biomechanical study. Spine (Phila Pa 1976) 2006;31:206-11.

5. Sairyo K, Sakai T, Mase Y, et al. Painful lumbar spondylolysis among pediatric sports players: a pilot MRI study. Arch Orthop Trauma Surg 2011;131:1485-9.

6. Yamazaki K, Kota S, Oikawa D, Suzuki Y. High defect stage, contralateral defects, and poor flexibility are negative predictive factors of bone union in pediatric and adolescent athletes with spondylolysis. J Med Invest 2018;65:126-30.

7. Sakai T, Goto T, Sugiura K, et al. Bony healing of discontinuous laminar stress fractures due to contralateral pars defect or spina bifida occulta. Spine Surg Relat Res 2018;3:67-70.

8. Fujii K, Katoh S, Sairyo K, Ikata T, Yasui N. Union of defects in the pars interarticularis of the lumbar spine in children and adolescents: the radiological outcome after conservative treatment. J Bone Joint Surg Br 2004;86:225-31. 
9. Tatsumura M, Gamada H, Ishimoto R, et al. Prevalence of curable and pseudoarthrosis stages of adolescent lumbar spondylolysis. J Rural Med 2018;13:1059.

10. Sakai T, Sairyo K, Mima S, Yasui N. Significance of magnetic resonance imaging signal change in the pedicle in the management of pediatric lumbar spondylolysis. Spine (Phila Pa 1976) 2010;35:E641-5.
11. Sairyo K, Sakai T, Yasui N. Conservative treatment of lumbar spondylolysis in childhood and adolescence: the radiological signs which predict healing. J Bone Joint Surg Br 2009;91:206-9.

12. Sairyo K, Katoh S, Sasa T, et al. Athletes with unilateral spondylolysis are at risk of stress fracture at the contralateral pedicle and pars interarticularis: a clinical and biomechanical study. Am J Sports Med 2005;33:583-90. 